\title{
Progress Towards the Investigation of Technical Issues Relevant to the Design of an Aircraft Wake Vortex Advisory System (WakeVAS)
}

\author{
David K. Rutishauser * \\ NASA Langley Research Center \\ Hampton, Virginia
}

\begin{abstract}
Wake vortex separations applied to aircraft during instrument operations have been shown to potentially introduce inefficiencies in air traffic operations during certain weather conditions conducive to short duration wake hazards between pairs of landing aircraft. NASA Langley Research Center (LaRC) demonstrated an integration of technologies that provided real-time observations and predictions of aircraft wake behavior, from which reduced wake spacing from the current criteria was derived. In order to take this proof of concept to an operational prototype system, NASA has been working in cooperation with the FAA and other government and industry members to design operational concepts for a Wake Vortex Advisory System (WakeVAS). In addition to concept development, open research issues are being addressed and activities to quantify system requirements and specifications are currently underway. This paper describes the technological issues relevant to WakeVAS development and current NASA efforts to address these issues.
\end{abstract}

\section{INTRODUCTION}

NASA Langley Research Center has a long history of contributions to aircraft wake vortex research, culminating with the demonstration of the Aircraft VOrtex Spacing System (AVOSS) at Dallas/Forth Worth International Airport in July $2000^{1}$. The AVOSS was a concept for an integration of technologies applied to providing dynamic wake-safe reduced spacing for single runway arrivals, as compared to current FAA separation standards applied during instrument operations. AVOSS included state-of-the-art weather sensors; wake sensors, and a wake behavior prediction algorithm. Using realtime data the AVOSS demonstrated an average 6\% potential throughput increase over current standards ${ }^{1}$. Since the AVOSS demonstration, two major program activities encompassing subsequent wake vortex work have developed: (1) NASA LaRC is currently supporting the Virtual Airspace Modeling and Simulation (VAMS) project at Ames Research Center as a concept developer for future aircraft wake vortex hazard/impact mitigation in the National Airspace System (NAS), and (2) NASA and the FAA are developing a (yet to be named) joint program for wake vortex research, described in a draft Research Management Plan (RMP). The VAMS project goals are to: (1) Develop advanced air transportation concepts, (2) Develop the capability to model and simulate behavior of these concepts to "never-beforeachieved" levels of fidelity, (3) To assess the concepts for impacts and effectiveness, and (4) To provide roadmaps for implementation of the concepts. The goal of the FAA/NASA RMP is to coordinate research and development efforts to realize operational solutions to wake vortex impacts on operations. The RMP breaks these activities into short (1-2 years), medium (3-5 years), and long (5-7 years) time horizons for the delivery of prototypes for solutions. This paper summarizes current and planned work under these two efforts, and open research questions and technology issues that must be addressed to successfully realize an operational wake vortex mitigation solution.

\section{CURRENT OPERATIONS}

Current wake vortex separations are achieved with a set of rules for air traffic control and procedures for pilots. The pilot procedures apply any time aircraft are operated under Visual Flight Rules (VFR) or on visual approaches under Instrument Flight Rules (IFR), and summarize safe operational practices based on a general understanding of wake behavior. In instrument meteorological conditions, pilots typically cannot see other aircraft and consequently under instrument operations the controller has the responsibility to provide wake separation to aircraft. The separation is achieved with a set of rules found in the Air Traffic Controller's Handbook $^{2}$, and shown in Table 1.

\footnotetext{
* Aerospace Technologist, Airborne Systems Competency
} 
Table 1 FAA Separation Rules

\begin{tabular}{|c|c|c|}
\hline $\begin{array}{l}\text { Type of Terminal } \\
\text { Operation }\end{array}$ & $\begin{array}{l}\text { Single Runway or } \\
\text { Parallel Runways } \\
\text { Less than } 2500 \mathrm{ft} \\
\text { Apart }\end{array}$ & Intersecting Runways \\
\hline Departures & $\begin{array}{l}\text { Behind B757 or heavy- } 120 \text { second hold; } \\
180 \text { seconds if intersection or opposite } \\
\text { direction same runway } \\
\text { OR } \\
\text { Radar separation minima } \\
\text { 1. Heavy behind heavy- } 4 \mathrm{mi} \\
\text { 2. Large/Heavy behind } \mathrm{B757}-4 \mathrm{mi} \\
\text { 3. Small behind B757 }-5 \mathrm{mi} \\
\text { 4. Large behind heavy }-5 \mathrm{mi} \\
\text { 5. Small behind heavy }-5 \mathrm{mi} \\
\text { For pairs not listed the separation is } 3 \\
\text { miles }\end{array}$ & $\begin{array}{l}120 \text { seconds behind B757 or heavy } \\
\text { departure or landing if projected flight } \\
\text { paths will cross; includes parallel } \\
\text { runways more than } 2500 \text { ft in } \\
\text { separation if will fly through the } \\
\text { airborne path of other aircraft }\end{array}$ \\
\hline Arrivals & $\begin{array}{l}\text { Radar separation minima (at threshold): } \\
\text { 1. Heavy behind heavy- } 4 \mathrm{mi} \\
2 . \text { Large/Heavy behind } \mathrm{B} 757-4 \mathrm{mi} \\
\text { 3. Small behind B757 }-5 \mathrm{mi} \\
4 \text {. Large behind heavy }-5 \mathrm{mi} \\
5 . \text { Small behind large }-4 \mathrm{mi} \\
6 . \text { Small behind heavy }-6 \mathrm{mi} \\
\text { For pairs not listed the separation is } 3 \\
\text { miles, except } 2.5 \text { miles in cases when } 50 \\
\text { second runway occupancy time is } \\
\text { documented and other criteria are met } \\
\text { Non-radar minima: } \\
\text { 120 seconds for aircraft landing behind } \\
\text { an arriving Heavy/B757, except if follower } \\
\text { is small then } 180 \text { seconds }\end{array}$ & $\begin{array}{l}120 \text { seconds for aircraft arriving after } \\
\text { a departing or arriving } B 757 \text { or heavy } \\
\text { if arrival will fly through the airborne } \\
\text { path of other aircraft }\end{array}$ \\
\hline
\end{tabular}

As shown in Table 1, the rules are based on leader/follower weight categories and are time or distance-based. The separations are applied during arrivals and departures to single runways and certain runway complexes. Much progress has been made in quantifying the wake behavior as influenced by atmospheric factors such as winds, turbulence and thermal stratification. Wake vortex avoidance rules that are sensitive to the dynamic influences on wake behavior could provide much more efficient spacing criteria than the worst-case criteria currently used. In support of both the VAMS and RMP projects, NASA Langley is developing concepts for minimizing the impact of aircraft wakes by applying the technologies demonstrated in
$\mathrm{AVOSS}^{3,4}$ in a general manner to address wake constraints at potentially any airport with any operational configuration $^{5}$.

\section{ENABLING TECHNOLOGIES}

The enabling technologies for a WakeVAS include each of the wake sensor systems ${ }^{6,7,8}$, and the wake prediction algorithm tested during $\mathrm{AVOSS}^{9}$. Some of the enabling technologies for the current WakeVAS concept were not demonstrated during AVOSS. All the potential WakeVAS technologies are listed as follows, with notes on their maturity level: 
1. Wake Sensors - The AVOSS utilized pulsed and CW Light Detection And Ranging (LIDAR) systems $^{6,7}$ for measurements of vortex location and strength. A windline ${ }^{8}$ was also used for measurements of vortex lateral position. Each sensor system used in AVOSS could be classified as a research sensor, but commercial pulsed LIDARs with wake-measuring capabilities can now be purchased. Detailed performance specifications of even the commercial LIDAR have yet to be determined. In addition, none of the AVOSS sensors could measure both wake position and strength in all weather conditions. Due to this and other limitations research continues on other candidate wake sensors.

2. Weather Sensors - AVOSS used a variety of commercial weather sensors to characterize the wake-relevant terminal area ambient conditions. A down select of the weather sensors used in AVOSS is required to determine the minimum necessary WakeVAS sensor suite. Candidates include an instrumented tower (for low-level wind, temperature, and turbulence measurements), a UHF profiler with a Radio Acoustic Sounding System (RASS) (low to middle level winds and temperature), a pulsed LIDAR (serving the dual task of wake and wind measurement), and aircraft measurements. Aircraft have the potential of measuring all the parameters of interest at a high resolution, under all weather conditions, and over the entire region of interest. Some corroboration with ground sensors is likely to still be required. A survey of the capabilities of current commercial weather sensors was performed in ${ }^{10}$.

3. Terminal Weather Predictor-A WakeVAS will cause dynamic changes to airport departure and arrival rates. In order for affected parts of the NAS to react and take advantage of the changes, sufficient advance knowledge of the changes will be required. This can be achieved with an accurate terminal-area-scale prediction of the relevant environmental parameters that affect wake behavior. A technology for accomplishing this was demonstrated in the AVOSS project, called the Terminal Area Planetary Boundary Layer Prediction System (TAPPS) ${ }^{11}$.

4. Sensor Fusing Algorithms - Data from a variety of sensors with different resolutions/effective ranges, and operational constraints will have to be integrated into single profiles of winds, temperature, and turbulence. Algorithms for fusing these sensor inputs (the sensor data often disagrees, as discovered during AVOSS) must be developed. These algorithms must include quality control measures so the confidence in the reported parameters can be determined. The AVOSS included a prototype for this function, see references ${ }^{3,4}$.

5. Wake Prediction Algorithms - The real-time wake behavior prediction algorithm used in $\mathrm{AVOSS}^{9}$ represents the state-of-the-art in a realtime wake model. Despite its sophistication it will not be adequate for an operational system because it does not specify the wake behavior in a probabilistic manner. A mean and variance of the wake position and strength is required along with a confidence measure of those values to perform a formal safety analysis of the system. The wake prediction algorithm should also be integrated with the weather predictions, observations, and wake observations in a closedloop system that adjusts for predictions diverging from observations. This configuration has not previously been tested.

6. Aircraft Meteorological Data-As mentioned in (2), aircraft may be the only sensing system with the capability to collect all the required environmental data over the region of interest. Aircraft already measure and report meteorological parameters, but the resolution of the data is not adequate for a WakeVAS. Data such as wind speed and direction can be corrupted by aircraft configuration changes (e.g. flap and gear operation) during the takeoff and landing phases of flight. This problem can be addressed with proper software processing of the data. The feasibility of obtaining the required resolution data from the aircraft systems has been demonstrated, but not in realtime.

7. Air/Ground Data Link - The concept requires both meteorological and aircraft state data (e.g. speed, weight) to be communicated to the ground prediction system. The bandwidth of the link is still an open research question.

8. Controller Tools/Displays - No controller tool was tested during the AVOSS project. The system was designed, however to interface through a dynamic set of weight-category 
dependent spacing standards to active approach spacing tools. A high-resolution spacing tool is one option, and at the other spacing resolution extreme is a wake-factor/no-factor with duration advisory, possibly displayed in a similar manner as the ITWS windshear alerts. The controller tool is an open design issue.

9. Flight Deck Displays - Similar to the controller tools, no flight deck displays for wake information have been tested; so many issues such as human factors for the design, symbology, coding, alerting and display location remain open research questions. A synthetic vision system is one candidate technology for displaying wake information. Another is a Cockpit Display of Traffic Information standalone display, or information integrated with the Navigation/Guidance/Multifunction display.
Figure 1 shows a proposed WakeVAS architecture using the aforementioned technologies. See ${ }^{5}$ for a description of the system concept and operation.

\section{RESEARCH ISSUES}

A variety of open research questions remain that prevent completely specifying a WakeVAS. They are listed with explanations in this section.

1. Accuracy/performance of all sensor subsystems - Where possible, each subsystem's performance should be represented in a probabilistic manner, such as a probability density function. This will facilitate systemlevel trade and safety analysis, and the generation of detailed subsystem specifications.

2. Development of probabilistic wake predictorSee the justification in (1). The lessons learned

\section{Figure 1 Proposed WakeVAS architecture}

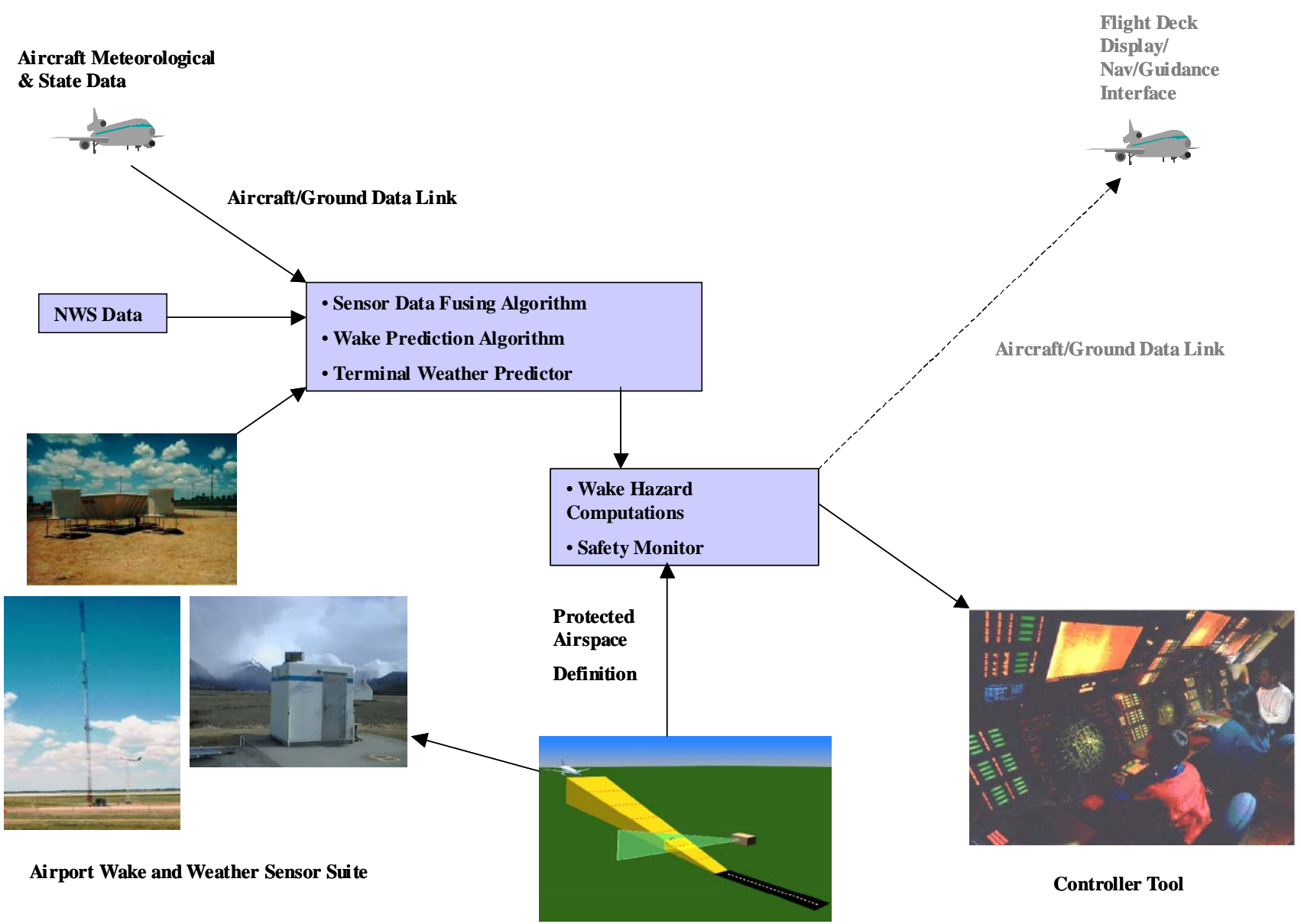


from the development of the AVOSS predictor will have to be applied to design a new predictor system with the required output.

3. Temporal and spatial variation of relevant weather parameters - These research questions have implications for the weather prediction horizon and the weather sensing requirements. The spatial (horizontal and vertical) variation of the meteorological parameters monitored will determine the required coverage and resolution of the ground and airborne weather sensors. The temporal variation impacts the measurement frequency of the weather sensors and the length of valid prediction intervals. The atmospheric variability around the terminal will be determined from meteorological observational field studies. WakeVAS development would get extra benefit from field studies since they provide data for the relevant meteorological parameters necessary to support the development of a statistical wake predictor and meteorological prediction models.

4. Safety analysis and rare event quantificationSince the proposed system will provide accurate wake hazard advisories it will need to meet a required level of safety through a formal safety analysis. All the non-normal and rare-normal events will need to be identified and analyzed as well.

5. Wake hazard definition - As mentioned in the system parameters section, a wake hazard metric needs to be defined for the CONOPS, based both on wake proximity and strength, and it should account for the response and size of encountering aircraft. A good deal of encounter analysis has been done in both the U.S. and Europe, but a technical and political consensus on what constitutes a wake hazard has yet to be agreed upon.

6. Quantification of weather prediction horizonAn open research question is the duration in which a terminal-scale weather prediction is valid, and how the confidence in the predictions should be reduced over time. The WakeVAS will have different NAS-level impacts depending on the amount of lead-time that exists prior to system changes.

7. Controller/Pilot workloads/Display design Many open research issues remain primarily in the human factors area regarding display design and the human interface for the WakeVAS. WakeVAS impacts on a controller's workload are not currently known, nor the impact wake information in the cockpit has on a pilot's situational awareness.

8. Data link requirements - The aircraft-to-ground data link requirements to support the WakeVAS concept need to be quantified. More information will need to be communicated at a higher frequency than is currently done for aircraft weather data, and links such as Automatic Dependent Surveillance Broadcast (ADS-B) do not include all the necessary WakeVAS parameters.

9. Lack of high resolution weather data - One major obstacle in performing cost/benefit studies for a WakeVAS concept is the current lack of the high resolution terminal area weather data needed to project ranges of wake behavior and the associated effectiveness of an active wake spacing system. Furthermore, the performance of statistical wake and meteorological prediction models is dependent on the quantity and quality of observed data. Research in obtaining this data from an effective combination of aircraft, field measurements, and weather models is currently active.

10. NAS impacts - As mentioned in (6), a WakeVAS will have system-level affects in the NAS, primarily by modulating airport acceptance rates. The impact of such a system is not currently known and will have to be studied via simulation.

\section{CURRENT RESEARCH ACTIVITIES}

Current work activities supported by the VAMS and RMP projects are described in this section. The work is targeted at the research issues described in the last section and at quantifying subsystem operation and highlevel requirements to support the process of designing WakeVAS specifications.

The AVOSS prediction algorithm requires a measure of the atmospheric turbulence to predict wake strength decay rates, and this measure has primarily been the Eddy Dissipation Rate (EDR). For AVOSS, EDR was measured with an instrumented tower, but the ability to obtain EDR from pulsed lidar wind measurements and from digital flight recorder data onboard an aircraft has 
been shown to be feasible. Investigations are underway to characterize the performance of these alternate means of measuring EDR.

In addition to investigating different means of sensing the required weather parameters, there is an effort to model the terminal area weather, similar to what was done for TAPPS (see Enabling Technology item 3). In addition to improving weather predictions, the goal of this effort is to create virtual weather input sets for locations where field measurements have not been taken to allow the simulation of WakeVAS implementations at any location of interest. This "virtual deployment" of a WakeVAS is useful for benefit studies (see Research Issues item 7).

There has been an ongoing activity to build a database for the large amount of wake measurements and associated weather data collected over the field deployments leading to the AVOSS demonstration. Over 10,000 wake measurements were taken, and several years of daily weather parameters down to a resolution of 1 minute are present. An online database is being developed so both researchers within NASA and others have an agile interface to the data to support analysis.

Work continues to design various WakeVAS concepts, assuming a phased implementation that employs increasing levels of technology and complexity to a variety of terminal operations. In order to estimate the benefits and impacts of the introduction of these new systems, behavioral models of the concepts are being developed leveraging off the AVOSS work. These models will be included in NAS fast-time simulations to support benefits/impacts studies.

To support the issues discussed in the Enabling Technologies section, an assessment is being conducted of the latest pulsed LIDAR wake detection and tracking algorithms (Item 1), and a study of the statistical behavior of the wakes from measured data (Item 5). Both of these activities fall under Research Issue (1), quantification of the performance of all WakeVAS subsystems. These activities are required to determine what the low-level subsystem specifications are, and if these meet high level system requirements.

\section{SUMMARY}

This paper provided an overview of current government and industry efforts NASA is leading to develop concepts and requirements for solutions to wake vortex capacity constraints on NAS operations. A list of the enabling technologies for NASA's current WakeVAS concept is provided with notes on each technology's maturity level.
Open research issues that limit the extent to which a WakeVAS can be specified are also identified.

\section{REFERENCES}

1 O'Connor, C., Rutishauser, D.; Enhanced Airport Capacity Through Safe, Dynamic Reductions in Aircraft Separation: NASA's Aircraft VOrtex Spacing System (AVOSS), Journal of Air Traffic Control, Volume 43, No. 3, October-December 2001, p. 4-10.

2 Air Traffic Controller's Handbook, FAA Order\# $7110.65 \mathrm{~N}$.

3 Hinton, D.; Description of Selected Algorithms and Implementation Details of a Concept-Demonstration Aircraft VOrtex Spacing System (AVOSS), NASA/TM2001-211027, June 2001.

4 Hinton, D., Charnock, J., Bagwell, D.; Design of an Aircraft Vortex Spacing System for Airport Capacity Improvement, AIAA 2000-0622, 38 ${ }^{\text {th }}$ AIAA Aerospace Sciences Meeting \& Exhibit, January 2000, Reno, NV. 5 Rutishauser, D., Lohr, G., Hamilton, D., Powers, R. McKissick, B., Adams, C., Norris, E.; Wake Vortex Advisory System (WakeVAS) Concept of Operations, NASA/TM-2003-212176, April 2003.

6 Campbell, S., Dasey, T., Freehart, R., Heinrichs, R., Matthews, M., Perras, G., Rowe. G.; Wake Vortex Field Measurement Program at Memphis, TN Data Guide, MIT Lincoln Laboratory Project Report NASA/L-2, 14 January 1997.

7 Britt, C.; Estimation of Aircraft Wake Vortex Characteristics from Coherent Pulsed Lidar Measurements, Research Triangle Institute Report RTI/4903/032-01F, January 1997.

8 Rudis, R., Burnham, D., Jacobs, L.; AVOSS Windline at Dallas/Ft. Worth Airport, Volume 1, Installation and Operation, DOT-VNTSC-RSPA-01-01, March 2001.

9 Robins, R., Delisi, D.; NWRA AVOSS Wake Vortex Prediction Algorithm Version 3.1.1, NASA/CR-2002211746, June 2002.

10 Zak, J.; Atmospheric Boundary Layer Sensors for Application in a Wake Vortex Advisory System, NASA/CR-2003-212175, April 2003.

11 Kaplan, Michael L.; Lin, Yuh-Lang; Charney, Joseph J.; Pfeiffer, Karl D.; Ensley, Darrell B.; DeCroix, David S.; and Weglarz, Ronald P.: A Terminal Area PBL Prediction System at Dallas-Fort Worth and its Application in Simulating Diurnal PBL Jets, Bulletin of the American Meteorological Society, Vol. 81, No. 9, September 2000. 